\title{
Incidencia del valor pragmático en la duración de la vocal acentuada del sufijo diminutivo -ito en el español de Chile
}

\author{
Ricardo Valderrama \\ Universidad Católica de la Santísima Concepción \\ Hernán E. Pérez* \\ Laboratorio de Fonética - Universidad de Concepción \\ Darío Fuentes \\ Universidad Católica de la Santísima Concepción
}

\begin{abstract}
Resumen
En este artículo, se presenta un estudio exploratorio de tipo cuantitativo que pretende determinar la influencia de la variable valor pragmático en la duración absoluta de la vocal acentuada (fonema/i/) presente en el sufijo apreciativo -ito del español de Chile. Con este propósito, se generó un protocolo en el que se daban, en contexto, múltiples realizaciones de palabras con el sufijo -ito en sus distintos valores pragmáticos. Mediante este protocolo, se obtuvo un corpus de 250 ítems léxicos con sufijos apreciativos diminutivos en contexto de habla controlada producidos por 10 hablantes nativos del español de Chile. Se midió en milisegundos la duración absoluta de la vocal
\end{abstract}

\footnotetext{
Para correspondencia, dirigirse a: Hernán Emilio Pérez (heperez@udec.cl), Universidad de Concepción, Departamento de Español, Laboratorio de Fonética, Casilla 160-C, Correo 3, Concepción, Chile.
} 
/i/ de cada una de las realizaciones del sufijo y se compararon los resultados mediante procedimientos estadísticos. Los resultados muestran que efectivamente existe incidencia del valor pragmático en la duración de la vocal acentuada en el sufijo diminutivo -ito del español de Chile.

Palabras clave: duración, valor pragmático, sufijo diminutivo, fonopragmática.

INFLUENCE OF THE PRAGMATIC VALUE IN THE ABSOLUTE LENGTH OF STRESSED Vowel of Diminutive SUfFiX -ITO IN Chilean Spanish

\begin{abstract}
A quantitative exploratory study that aims to determine the influence of the pragmatic value variable in the absolute length of stressed vowel (phoneme / i /) present in appreciative suffix -ito in Chilean Spanish is presented in this article. A protocol with several samples of the different pragmatic uses of the diminutive suffix in context was created. Using this protocol, a corpus of 250 lexical items with appreciative diminutive suffixes in context of controlled speech produced by 10 native Chilean Spanish speakers was obtained. Absolute length of the vowel /i/ for each token of the suffix was measured in milliseconds and the results were compared by statistical procedures. The results show that indeed there is an incidence of pragmatic value in length of the stressed vowel in Chilean Spanish diminutive suffix -ito.
\end{abstract}

Keywords: absolute length, vowel length, pragmatic value, diminutive suffix, phono-pragmatic.

Recibido: 01/08/16 Aceptado: 10/07/17

\title{
1. INTRODUCCIÓN
}

En este artículo, se presenta un estudio exploratorio de tipo cuantitativo que pretende determinar la influencia de la variable valor pragmático en la duración absoluta de la vocal acentuada (fonema /i/) presente en el sufijo apreciativo -ito del español de Chile. Autores clásicos ya han sugerido hace algunas décadas la posible relación entre el alargamiento temporal de la vocal tónica del sufijo en cuestión y el valor pragmático que éste porta, cuando 
los hablantes le asignan otros valores de sentido más allá del meramente diminutivo. Esta idea ya sugerida, más la observación de lo que ocurre en los hechos lingüísticos cotidianos, han llevado a los autores de este artículo a plantearse la necesidad de explorar de manera empírica este fenómeno.

Estudios recientes han llegado a determinar que existen al menos cuatro valores pragmáticos, además del diminutivo, que los hablantes le dan en el uso a este sufijo. Tomando como referencia esta categorización, se generó un corpus de habla controlada en el cual se daban, en contexto, múltiples realizaciones de palabras con el sufijo -ito en sus distintos valores pragmáticos. Se midió en milisegundos la duración absoluta de la vocal/i/ de cada una de las realizaciones del sufijo y se compararon los resultados mediante procedimientos estadísticos.

Esta investigación pretende aportar al conocimiento del comportamiento de los elementos prosódicos del idioma frente a realizaciones pragmáticas de elementos de uso cotidiano.

\section{MARCO CONCEPTUAL}

\subsection{SUFIJO APRECIATIVO DIMINUTIVO}

El sufijo apreciativo diminutivo está presente en casi todas las situaciones comunicativas de la vida cotidiana, siendo reconocido regionalmente según la opinión popular como un elemento cultural propio de los chilenos. Sin embargo, a pesar de ser un elemento que parece estar tan arraigado en la cultura, no ha habido mucha investigación sobre esto en Chile y la que existe se centra en su uso pragmático (Quintrileo 2001) o en su aplicación y uso en el campo de la traducción (Valderrama 2010), sin considerar, hasta el momento, otros aspectos lingüísticos como el fonético, ni, más específicamente, la influencia que podría tener especialmente el componente prosódico en sus realizaciones pragmáticas.

Varela (2005: 8) afirma que "[1]a lengua se vale de procedimientos morfológicos para la formación de palabras; el resultado de estos procedimientos son las 'palabras complejas'. Estas se forman, fundamentalmente a través de dos tipos de procesos: la 'derivación' mediante afijos (prefijos y sufijos) y la 'composición"”. También agrega que (41) “[...] la derivación léxica mediante sufijación es el procedimiento de formación de palabras más productivo, general y variado de nuestra lengua", por lo tanto, es indudable que los sufijos son fundamentales en el español, ya que 
a través de ellos es posible enriquecer los componentes morfosintáctico y semántico de la lengua. Seco (1994) distingue entre dos tipos de sufijos, los que modifican la palabra gramaticalmente y aquellos que entregan un nuevo valor semántico, estos últimos son conocidos como los sufijos apreciativos.

\subsection{Polivalencia del Diminutivo}

El sufijo apreciativo diminutivo tiene diferentes funciones y valores que dependen del acto de habla. Searle (1969) plantea que hablar una lengua es una forma de conducta que es gobernada por reglas y que estas reglas son condiciones necesarias para la ejecución de tipos particulares de actos de habla. Es, precisamente, dentro de este marco que Reynoso (2005) señala que:

Aparentemente, el uso del diminutivo en español responde sólo a necesidades de variación semántica y estilística. Sin embargo, el análisis del uso de esta marcación permite observar regularidades tanto en la conformación de la escena discursiva donde se echa mano del diminutivo cuanto en las intenciones comunicativas que establece el emisor. El uso de un diminutivo permite al hablante crear contextos comunicativos siempre novedosos y, sobre todo, altamente idiosincrásicos, pues su uso parece estar regulado culturalmente (79).

Hummel (1997: 195), sobre el carácter de los diminutivos que van más allá de lo meramente morfológico-gramatical, afirma: “[...] 1, cada palabra que se combina con un sufijo diminutivo se realza gracias a este sufijo;" y lo más interesante en este caso " 2 , el oyente/lector debe reparar en una apreciación especial cuyo carácter depende del contexto y la situación".

\subsection{VALORES PRAGMÁTICOS DEL APRECIATIVO DIMINUTIVO}

El sufijo apreciativo diminutivo, como ya se ha mencionado, posee diferentes funciones y valores. Valderrama (2010: 9), al estudiar y proyectar este fenómeno en el trabajo de la traducción, establece que "[l]a función del diminutivo, no es solamente la de empequeñecer una unidad. El diminutivo destaca por su versatilidad y puede cumplir diferentes funciones, dependiendo del contexto en el que se encuentre y de la actitud del hablante sobre el objeto o receptor". Estas funciones, según Quintrileo (2001) son tres: la función de apreciación cuantitativa (valor diminutivo propiamente tal); la función de apreciación cualitativa emocional (se refiere al valor afectivo 
o despectivo) y la función de apreciación cualitativa no emocional (en esta función destacan los valores de intensificación, cortesía, atenuación, ironía, etc.). Quintrileo estudió un corpus de 625 usos de diminutivo. Estos usos se elicitaron principalmente de discursos de los medios de comunicación masiva, tales como locuciones radiales, programas de televisión y entrevistas telefónicas en la radio y corresponden en su mayoría a conversaciones de tono informal. Quintrileo separó el corpus y clasificó los usos de los diminutivos según la función pragmática que en cada situación a estos se les asignaba. Entre los usos con mayor frecuencia de aparición, la autora destaca los de valor afectivo, activo, intensificador, atenuante y diminutivo.

El valor más ampliamente conocido del sufijo apreciativo diminutivo, o por lo menos el que siempre primero se menciona, es precisamente este uso que le da nombre al sufijo. La RAE (2015) define, de hecho, al sufijo diminutivo como aquél "[q]ue denota disminución de tamaño en el objeto designado". Alonso (1951: 197) lo define como el que tiene "significación de empequeñecimiento", sin embargo, pone en duda que este valor sea el único, $\mathrm{y}$ considera a este uso como el menos frecuente, debido a que muchas veces, por ejemplo, el valor del diminutivo es intensificado por otra palabra como en "cajita pequeña". Es más, sobre esto último, Hummel (1997) sostiene que el significado diminutivo no se encuentra realmente en los sufijos diminutivos sino que en los adjetivos postnominales.

No obstante, el uso del sufijo diminutivo con este valor es un hecho en la lengua, como se ilustra en los siguientes ejemplos:

(1) Él vivía en una casita chiquitita.

(2) ¿Dónde están las esponjitas?

(3) Ahí, en el cajoncito chico.

Sobre el valor activo del sufijo diminutivo, Quintrileo señala que

[...] existe un empleo del diminutivo que busca influir en el pensamiento del oyente, intenta persuadir, convencer, etc., esto es adoptan incluso un carácter retórico $[\ldots]$ este valor será tal vez más frecuente en actos de habla tales como las peticiones, órdenes, e incluso las invitaciones, los cuales actúan más directamente sobre el oyente (2001: 28).

En este valor, predomina la función conativa del lenguaje. El uso del sufijo diminutivo con valor activo se puede ilustrar con los siguientes ejemplos:

(4) Ahora te me quedas calladito y haces todas tus tareas.

(5) Pero, papito, quiero salir a jugar. 
Alonso (1951) destaca además el carácter afectivo de los diminutivos, que es la actitud que tiene el emisor sobre lo que nombra o escribe. Para Quintrileo (2001), el valor afectivo del diminutivo se subdivide también en distintas funciones, como la de compasión, la de burla, la de cariño y la despectiva. Se entiende entonces el valor afectivo del diminutivo como aquel que representa una apreciación cualitativa que el emisor tiene emocionalmente con el objeto, sea esta positiva o negativa. Este uso se da con frecuencia cuando se le asigna el sufijo diminutivo a los nombres propios. Se puede apreciar el uso con valor afectivo en los siguientes ejemplos:

(6) Mi viejito está muy bien, gracias.

(7) ¿Has visto a Jorgito?

(8) Luchito, ¿me pasas esa caja, por favor?

Seco (1994) señala que a pesar del nombre que posee, el diminutivo es muchas veces intensificador. Algo interesante plantea Alonso (1951) cuando señala que es en este valor donde existe un alargamiento en la duración de la vocal acentuada y que es precisamente esto lo que permite al hablante identificar su valor pragmático.

El uso del diminutivo con valor de intensificador se puede observar en estos ejemplos:

(9) El agua esta clarita.

(10) Después de estos días fríos, hoy se siente mucho más calentito.

Pandolfi (1989: 38) establece que el valor atenuante del diminutivo tiene la función de "atenuar la característica negativa que se le atribuye a una entidad léxica sustantiva", por ejemplo, cuando se dice que alguien está "gordito" con la intención de que esto no se sienta o interprete como algo negativo u ofensivo. Quintrileo (2001: 26) señala al respecto que el diminutivo "actúa indirectamente evitando la explicitación de algo que resulta penoso".

El uso del diminutivo con valor atenuante se puede ver, por ejemplo, en enunciados como:

(11) Dicen que ella es medio feita.

(12) Estuvo un poquito enfermito.

(13) Siempre me dicen que estoy gordito.

Desde una dimensión pragmática, los valores del diminutivo tienen relación con el contexto comunicativo en el que los interlocutores se encuentran inmersos y la intención que los hablantes quieren declarar. En consecuencia, es evidente que el sufijo diminutivo no solo se manifiesta con este valor, 
que puede considerarse como el no marcado, sino que dependiendo del contexto y de la intención comunicativa asume otros valores que ya han sido documentados y que pueden ser ilustrados mediante ejemplos.

\subsection{LA FonOPRAGMÁTICA}

Herrero (2006: 21) declara que la pragmática dentro del área de la lingüística "[...] estudia la producción y la interpretación de los enunciados, es decir el uso de la lengua por hablantes en los actos y en las situaciones de comunicación". No debiera extrañar entonces que exista un espacio común de estudio entre dos disciplinas como la fonética y la pragmática, una disciplina que intente explicar cómo un finito número de recursos fónicos segmentales y prosódicos pueden añadir al mensaje nuevos matices de sentido.

Si bien es cierto, es posible encontrar investigación sobre los componentes morfológicos, semántico y pragmáticos de los diminutivos, hasta ahora no ha sido posible encontrar evidencia empírica acerca de si los elementos suprasegmentales de la lengua son los que permiten establecer las diferencias entre una función y otra de este. De hecho, los estudios sobre los correlatos acústicos parecen ser algo relativamente nuevo en Chile. Destacan entre estos (aunque relativos a otros temas, no específicamente acerca del diminutivo) el trabajo realizado por Roldán (2000) sobre los correlatos acústicos en la atenuación en Chile o el de Pereira (2011) sobre la entonación de algunos marcadores discursivos del español de Chile relacionados con patrones de enfado, asentimiento y extrañeza; en su investigación Pereira concluye que la variación en la configuración fonética suprasegmental de los marcadores que estudia está asociada con la variación del significado pragmático.

Estas investigaciones se enmarcan en el área de lo que Hidalgo considera los albores de una nueva disciplina:

Algunos investigadores han propuesto desarrollar una disciplina especializada en la descripción de las funciones entonativas relacionadas con este tipo de valores, visibles también en otros ámbitos del componente fónico de las lenguas (el segmental y el paralingüístico, al menos); esta disciplina ha recibido la denominación "provisional" de fonopragmática (2006: 34).

La relación que se puede establecer entre las funciones y los elementos prosódicos de la lengua es que ambos tienen relación con la semántica. Fernández Planas (2005) establece que la información semántica de las palabras se encuentra en la prosodia y que (52) "los rasgos suprasegmentales o prosódicos son tres: el acento, el tono (o la sucesión de ellos, la entonación) 
y la duración". De la misma manera Fernández Planas hace hincapié en cómo los suprasegmentos son muchas veces los que llevan la información codificada desde la pragmática.

Se ha demostrado también cómo otros rasgos suprasegmentales como la entonación son un recurso fundamental cuando se atenúa en el español de Chile (Roldán 2000) o cómo la duración vocálica tiene estrecha relación con el nivel social y su procedencia geográfica (Pereira y Soto-Barba 2011).

\subsection{La Duración}

En cuanto a la duración, a veces también llamada cantidad, Fernández Planas la define como:

parámetro segmental y suprasegmental que consiste en el mantenimiento en el tiempo de una determinada configuración articulatoria [...] Como elemento suprasegmental, la duración, además de ser mayor en las sílabas tónicas, se hace especialmente evidente en las que constituyen el tonema (o núcleo final de las frases desde la última tónica) puesto que, al margen de la modalidad de la oración, puede aumentar considerablemente respecto a las que forman el pretonema (cuerpo de la frase antes del tonema) (2005:55).

Martínez Celdrán (1998) sostiene que la duración es uno de los índices de la tensión que poseen los sonidos, esto debido a que la tensión exige un mayor esfuerzo articulatorio y esto conlleva a una mayor cantidad de tiempo para realizarlo.

Sobre la duración de las vocales, Martínez Celdrán (1998) establece que esta depende del tipo de sílaba (tónica o átona) en la que se encuentren, pero que esto no constituye una diferencia significativa. Por su parte, Quilis y Fernández (1969) explican que la duración de las vocales en español no tiene relevancia fonológica y que desde el punto de vista práctico deben ser consideradas como breves. Martínez Celdrán (1998) describe las vocales y dice que la duración de cada sonido depende de su naturaleza, asimismo señala que el contexto también influye en la duración, por ejemplo, las vocales son más largas cuando anteceden a las oclusivas sonoras.

Sin embargo, si bien es cierto que la cantidad vocálica, o duración, no es un elemento fonológicamente relevante, esto no quiere necesariamente decir que semántica o pragmáticamente no lo sea. De hecho, Pereira y Soto-Barba (2011) establecen en una investigación sobre las vocales de la provincia de Ñuble que la duración de éstas podría estar vinculada con variables pragmáticas. En su investigación observaron cómo el estrato social 
influye en la cantidad vocálica: mientras el hablante se encontraba más cercano a la ciudad y más alto era su estrato social, menor era la duración de sus vocales y viceversa. Entre las posibles explicaciones a este fenómeno están las relacionadas con la mayor o menor tensión articulatoria, que ha sido considerada como factor que puede modificar la cantidad vocálica, pero también está relacionada con factores extralingüísticos como la concepción del tiempo de los informantes y el grado de familiaridad con la situación comunicativa establecida para obtener las grabaciones.

Marín (1994-1995) establece sobre la duración de las vocales que:

[1]a posición que ocupa un sonido en la frase es un factor que influye en su duración. Los resultados muestran la existencia de una correlación entre posición prepausal y una mayor duración de la vocal [...] La media de duración de las vocales en posición prepausal es de 94,51 ms. Este valor medio contrasta con el de las no prepausales, que se sitúa en 64,29 ms. Ello representa un incremento del 47\% (222).

Marín (1994-1995) y Hely (1996-1997) realizan sendos estudios sobre la duración de las vocales en el español y coinciden en algunos de los factores que podrían influir en la cantidad vocálica: posición final/no final en la palabra, condición acentuada/inacentuada, tipo silábico, consonante siguiente, número de segmentos en la sílaba, estructura silábica, posición con respecto a la sílaba acentuada, número de segmentos de la palabra, número de sílabas de la palabra, duración de la sílaba y duración de la palabra. Sin embargo, la intención pragmática no se aprecia dentro de los posibles factores que podrían condicionar la cantidad vocálica.

A partir de todos los antecedentes ya expuestos, es posible preguntarse, entonces, si el valor pragmático del sufijo apreciativo diminutivo incide, efectivamente, en la cantidad vocálica del fonema /i/. ¿Es plausible la aseveración de Alonso (1951) de que el valor intensificador produce alargamiento en la vocal tónica del sufijo apreciativo? Y esto, de ser cierto, ¿es lo que lo distingue de sus demás valores pragmáticos? Dicho de otro modo, ¿es la duración de la vocal tónica un factor determinante en la producción y, por consecuencia, en la percepción e interpretación del valor pragmático de un diminutivo? 


\section{OBJETIVOS}

\subsection{Objetivo General}

Determinar la influencia del valor pragmático en la duración de la vocal acentuada del sufijo apreciativo diminutivo.

\subsection{OBJETIVOS ESPECÍFICOS}

- Medir la duración absoluta del fonema /i/ en cada uno de los valores pragmáticos del sufijo apreciativo diminutivo.

- Comparar los distintos valores de la duración de la vocal acentuada del sufijo apreciativo en sus distintos valores pragmáticos.

\subsection{Hipótesis}

$\mathrm{H}_{1}$. La duración de la vocal /i/ del sufijo -ito es mayor en el valor intensificador que en otros valores pragmáticos.

$\mathrm{H}_{2}$. La duración de la vocal /i/ del sufijo -ito se verá modificada por el valor pragmático que el emisor dé a su mensaje.

\section{MARCO METODOLÓGICO}

\subsection{TIPO DE INVESTIGACIÓN}

La investigación que aquí se expone es de carácter cuantitativo, descriptivo, exploratorio y transversal.

\subsection{Materiales y MÉTOdos}

Se elaboró un protocolo para la grabación de los audios (ver anexo 1). El protocolo consistía en un libreto para ser leído que constaba de 4 diálogos y un texto. Dentro del protocolo se contextualizaron 25 palabras que contenían el sufijo apreciativo con diferentes valores pragmáticos. 


\subsection{VALIDACIÓN DEL INSTRUMENTO}

Debido a que, en un inicio, el protocolo fue elaborado solo por el investigador principal, con el propósito de verificar si el valor pragmático asignado a cada realización del diminutivo era el adecuado, previamente a la grabación y obtención del corpus, se confeccionó una encuesta de validación del instrumento (Anexo 2), cuyo propósito es medir la apreciación subjetiva de distintas personas y su interpretación del uso del sufijo diminutivo en cada caso.

Esta encuesta fue aplicada a dos grupos, en el primero participaron 30 sujetos (15 hombres y 15 mujeres), promedio de edad de 27,5 años (SD 7,24) de diversas ocupaciones, sin conocimientos de lingüística (hablantes nativos comunes). En el segundo grupo participaron 7 mujeres con un promedio de edad de 48 años $($ SD 8,124) con la característica particular de ser especialistas en lingüística (todas con estudios de posgrado en la disciplina).

A los encuestados se les solicitó leer en silencio los 4 diálogos y el texto que contenían las palabras con los sufijos apreciativos en contexto y se les pidió clasificar la función del sufijo según su interpretación de la intención del emisor, pudiendo elegir solo una opción entre: valor diminutivo, afectivo, intensificador, activo y atenuante. La información obtenida de esta encuesta se comparó con los valores asignados por el autor del protocolo. Y a partir de esta comparación se decidió el valor pragmático final que se le asignaría a cada uno de los diminutivos que formaban parte del protocolo.

En algunos de los casos se obtuvo un 100\% de acuerdo entre las opiniones de los hablantes comunes, los expertos en lingüística y el investigador; a estos casos se les denominó casos fuertes. A aquellos casos en que existe acuerdo entre dos grupos, se les denominó casos medios. Aquellos casos en donde existe discrepancia entre los tres grupos fueron calificados como casos débiles. Los resultados de la encuesta muestran que en el protocolo existen 14 casos fuertes (4 diminutivos, 4 atenuantes, 4 afectivos, 1 intensificador y 1 activo), 9 casos medios (4 intensificadores, 2 activos, 1 afectivo, 1 atenuante y 1 diminutivo) y 2 casos débiles ( 2 activos). Para resolver en los casos débiles se optó por asignarles la interpretación que fuese más frecuente entre los encuestados. El detalle completo de los resultados de la encuesta de validación del instrumento está disponible para ser consultado en el Anexo 3.

\subsection{INFORMANTES}

Una vez validado el protocolo, se procedió a hacer las grabaciones. Diez personas participaron como informantes en la grabación de los diálogos, 
hombres, promedio de edad 21,3 (SD 3,8), estudiantes de educación superior, nativos del español de Chile, de zonas urbanas y sin antecedentes de problemas de lenguaje.

Las grabaciones fueron capturadas utilizando un micrófono condensador USB Samson C01, con el software Audacity v 2.0.4 en un computador portátil con sistema operativo Windows 7. Estas fueron realizadas en dos lugares diferentes de grabación, ambos con salas de aislación acústica especialmente diseñadas para la grabación de audio. El primer lugar fue la sala de grabación de la Escuela de Comunicación de DUOC UC Concepción y el segundo fue el Laboratorio de Fonética del Departamento de Español de la Universidad de Concepción.

Finalmente, se obtuvo un corpus de 250 ítems léxicos con sufijos apreciativos diminutivos en contexto de habla controlada producidos por 10 hablantes nativos del español de Chile.

\subsection{Procedimientos}

Los audios fueron rotulados y editados utilizando el programa Praat v5.3.55. Se obtuvo la duración absoluta en milisegundos de la vocal tónica /i/ en cada una de las realizaciones como se ilustra en la Figura 1. Si bien es cierto, existe cierta controversia acerca de cuál es el parámetro más adecuado para medir la duración absoluta de un segmento, en este caso se decidió utilizar como referencia la aparición en el espectrograma del segundo formante. Los valores obtenidos se ordenaron y clasificaron de acuerdo con sus valores pragmáticos.

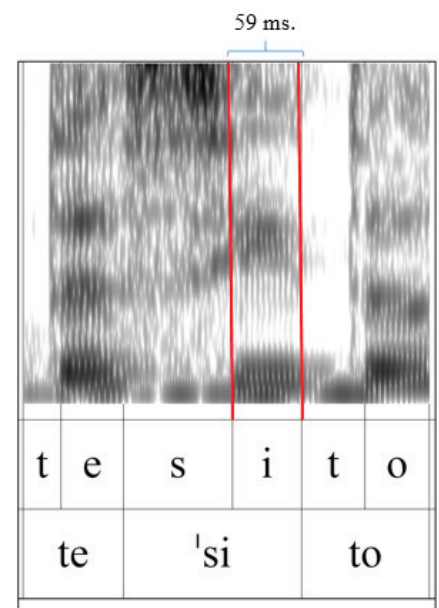

Figura 1. Espectrograma de la palabra "tecito" 
Los valores fueron ingresados al software de análisis estadístico $S P S S$ v.20, en el cual se realizó una tabla con los descriptivos y un ANOVA con prueba de Tukey, que permite establecer diferencias significativas entre dos o más grupos.

\subsection{IDENTIFICACIÓN DE LAS VARIABLES}

Como variable dependiente se ha definido la duración absoluta de la vocal /i/ expresada en milisegundos. Como variable independiente se ha establecido el valor pragmático del sufijo apreciativo diminutivo y sus variantes son valor diminutivo, activo, atenuante, afectivo e intensificador.

\section{RESULTADOS}

\subsection{DURACIÓN Y VALOR PRAGMÁTICO}

En total se obtuvieron 5 realizaciones por informante para cada valor pragmático, es decir, existen 50 realizaciones de /i/ para cada valor pragmático.

\begin{tabular}{|c|c|c|c|c|c|c|c|c|}
\hline & \multirow[t]{2}{*}{$\mathrm{N}$} & \multirow[t]{2}{*}{ Media } & \multirow{2}{*}{$\begin{array}{l}\text { Desv. } \\
\text { Std. }\end{array}$} & \multirow{2}{*}{$\begin{array}{l}\text { Error } \\
\text { Std. }\end{array}$} & \multicolumn{2}{|c|}{$\begin{array}{c}\text { Intervalo de } \\
\text { confianza } \\
(95 \%)\end{array}$} & \multirow[t]{2}{*}{ Min. } & \multirow[t]{2}{*}{ Max. } \\
\hline & & & & & $\begin{array}{l}\text { Límite } \\
\text { inferior }\end{array}$ & $\begin{array}{l}\text { Límite } \\
\text { superior }\end{array}$ & & \\
\hline Diminutivo & 50 & 58,68 & 13,263 & 1,876 & 54,91 & 62,45 & 38 & 107 \\
\hline Activo & 50 & 85,94 & 20,024 & 2,832 & 80,25 & 91,63 & 48 & 143 \\
\hline Atenuante & 50 & 78,24 & 21,372 & 3,022 & 72,17 & 84,31 & 34 & 171 \\
\hline Afectivo & 50 & 74,12 & 27,369 & 3,871 & 66,34 & 81,90 & 41 & 189 \\
\hline Intensificador & 50 & 75,64 & 15,044 & 2,128 & 71,36 & 79,92 & 48 & 101 \\
\hline Total & 250 & 74,52 & 21,794 & 1,378 & 71,81 & 77,24 & 34 & 189 \\
\hline
\end{tabular}

Tabla 1. Descriptivos de duración de vocal /i/ según valor pragmático (valores en milisegundos) 
Como puede apreciarse en la Tabla 1, el valor pragmático diminutivo presenta el menor valor promedio de duración así como también la menor desviación estándar de los grupos, lo que lo convierte en el grupo más homogéneo de todos. El valor activo, por su parte, presenta el mayor promedio de duración del fonema /i/. Entre los valores atenuante, afectivo e intensificador, se observa cierto grado de similitud en el promedio de la duración, sin embargo, la desviación estándar es mayor en el valor atenuante y especialmente en el afectivo, siendo este último el valor más alto de los grupos estudiados.

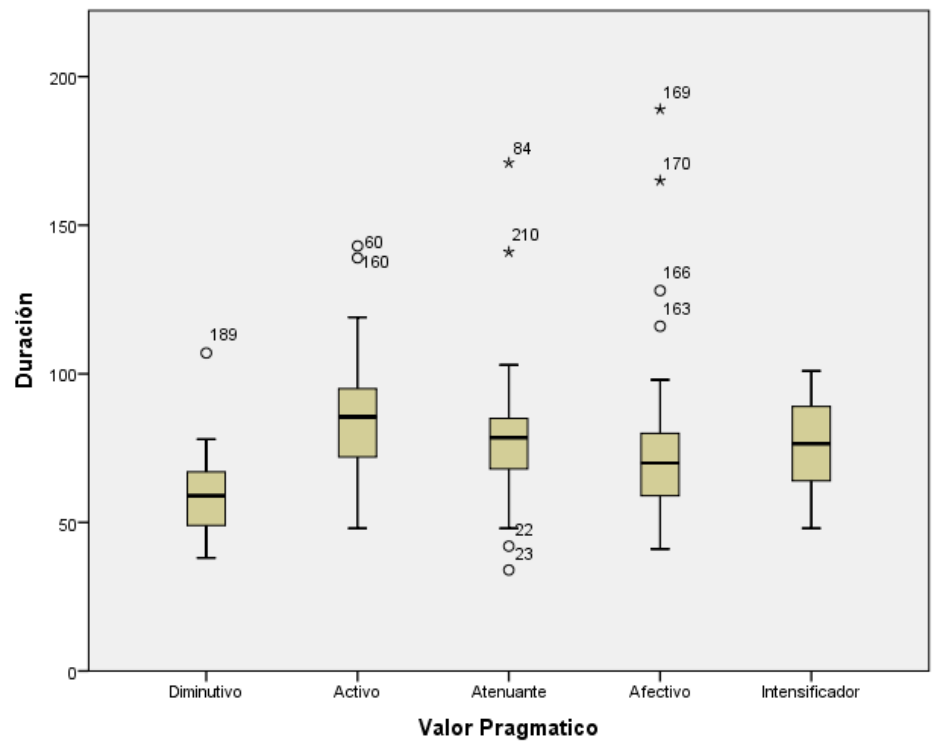

Gráfico 1. Distribución de los valores de duración de la vocal /i/ según valor pragmático

El Gráfico 1 nos presenta la distribución de los distintos valores de duración de la vocal según su valor pragmático. Los datos del valor diminutivo presentan cierta tendencia de concentración de valores menores a la mediana, con un grado de dispersión de datos menor que otros valores y presentando solo un caso atípico. El valor afectivo, por su parte, es el que se presenta de forma más irregular en comparación con los otros grupos estudiados; obtiene la mayor cantidad de casos atípicos y junto con el valor activo es el que presenta la mayor dispersión de los datos.

En la Tabla 2 se presentan los resultados del análisis $A N O V A$, con las comparaciones múltiples entre cada valor pragmático. Las diferencias significativas al .005 entre los grupos son señaladas con un asterisco. 
Variable dependiente: duración

\begin{tabular}{|c|c|c|c|c|c|c|}
\hline \multirow{2}{*}{$\begin{array}{c}\text { (I) Valor } \\
\text { Pragmático }\end{array}$} & \multirow{2}{*}{$\begin{array}{c}(\mathrm{J}) \text { Valor } \\
\text { Pragmático }\end{array}$} & \multirow{2}{*}{$\begin{array}{l}\text { Diferencia } \\
\text { entre } \\
\text { promedios } \\
\quad(\mathrm{I}-\mathrm{J})\end{array}$} & \multirow{2}{*}{$\begin{array}{l}\text { Error } \\
\text { Std. }\end{array}$} & \multirow{2}{*}{ Sig. } & \multicolumn{2}{|c|}{$\begin{array}{c}\text { Intervalo de } \\
\text { confianza } 95 \%\end{array}$} \\
\hline & & & & & $\begin{array}{r}\mathrm{L} . \\
\text { inferior }\end{array}$ & $\begin{array}{r}\text { L. } \\
\text { superior }\end{array}$ \\
\hline \multirow{4}{*}{ Diminutivo } & Activo & $-27,260^{*}$ & \multirow{20}{*}{4,009} &, 000 & $-38,28$ & $-16,24$ \\
\hline & Atenuante & $-19,560^{*}$ & &, 000 & $-30,58$ & $-8,54$ \\
\hline & Afectivo & $-15,440^{*}$ & &, 001 & $-26,46$ & $-4,42$ \\
\hline & Intensificador & $-16,960^{*}$ & &, 000 & $-27,98$ & $-5,94$ \\
\hline \multirow{4}{*}{ Activo } & Diminutivo & $27,260^{*}$ & &, 000 & 16,24 & 38,28 \\
\hline & Atenuante & 7,700 & &, 309 & $-3,32$ & 18,72 \\
\hline & Afectivo & $11,820^{*}$ & &, 029 &, 80 & 22,84 \\
\hline & Intensificador & 10,300 & &, 079 &,- 72 & 21,32 \\
\hline \multirow{4}{*}{ Atenuante } & Diminutivo & $19,560^{*}$ & &, 000 & 8,54 & 30,58 \\
\hline & Activo & $-7,700$ & &, 309 & $-18,72$ & 3,32 \\
\hline & Afectivo & 4,120 & &, 842 & $-6,90$ & 15,14 \\
\hline & Intensificador & 2,600 & &, 967 & $-8,42$ & 13,62 \\
\hline \multirow{4}{*}{ Afectivo } & Diminutivo & $15,440^{*}$ & &, 001 & 4,42 & 26,46 \\
\hline & Activo & $-11,820^{*}$ & &, 029 & $-22,84$ &,- 80 \\
\hline & Atenuante & $-4,120$ & &, 842 & $-15,14$ & 6,90 \\
\hline & Intensificador & $-1,520$ & & ,996 & $-12,54$ & 9,50 \\
\hline \multirow{4}{*}{ Intensificador } & Diminutivo & $16,960^{*}$ & &, 000 & 5,94 & 27,98 \\
\hline & Activo & $-10,300$ & &, 079 & $-21,32$ & ,72 \\
\hline & Atenuante & $-2,600$ & &, 967 & $-13,62$ & 8,42 \\
\hline & Afectivo & 1,520 & & ,996 & $-9,50$ & 12,54 \\
\hline
\end{tabular}

Tabla 2. Comparación de la duración de la vocal entre valores pragmáticos

Se observa, en la Tabla 2, que el valor diminutivo se diferencia del resto de manera significativa obteniendo la menor duración de los grupos comparados. El valor activo se diferencia significativamente de los valores afectivo y diminutivo, pero se asemeja a los valores intensificador y atenuante. El valor 
atenuante se diferencia significativamente solamente del valor diminutivo. Por su parte, el valor afectivo se diferencia de los valores diminutivo y activo. Finalmente el valor intensificador se diferencia solamente del valor diminutivo. Una explicación más sintética de estos resultados es observable en la Tabla 3.

\begin{tabular}{|l|c|c|c|c|}
\hline \multirow{2}{*}{$\begin{array}{c}\text { Valor } \\
\text { Pragmático }\end{array}$} & \multirow{2}{*}{$\mathrm{N}$} & \multicolumn{3}{c|}{$\begin{array}{c}\text { Subset para } \\
\text { alpha }=0.05\end{array}$} \\
\cline { 3 - 5 } & & 1 & 2 & 3 \\
\hline Diminutivo & 50 & 58,68 & & \\
\hline Afectivo & 50 & & 74,12 & \\
\hline Intensificador & 50 & & 75,64 & 75,64 \\
\hline Atenuante & 50 & & 78,24 & 78,24 \\
\hline Activo & 50 & & & 85,94 \\
\hline Sig. & & 1,000 &, 842 &, 079 \\
\hline
\end{tabular}

Tabla 3. Diferencias significativas de la duración entre valores pragmáticos

En la Tabla 3 se puede ver que los valores que se asemejan aparecen agrupados en una misma columna. Es así como se puede observar que el valor diminutivo se diferencia significativamente de todos los otros y a la vez se puede ver que entre el valor afectivo y el activo existe una diferencia levemente significativa.

\section{CONCLUSIONES}

Los resultados del proceso de validación del instrumento muestran que existe cierta dificultad por parte de los hablantes al momento de identificar el valor activo del sufijo diminutivo, esto se ve reflejado también en la irregularidad de las realizaciones en los textos por parte de los informantes.

Destaca además la fortaleza del valor diminutivo del sufijo, el cual fue reconocido en el mayor número de veces. Entonces, si bien es cierto lo que dice Quintrileo (2001) que el valor diminutivo no es el valor principal del sufijo, puesto que su frecuencia de uso no es tan alta como se podría pensar, se debe reconocer que los hablantes identifican claramente este valor por sobre otros, quizás apoyado esto por la vieja creencia de que el diminutivo se usa solo con este único valor semántico. 
En el valor diminutivo, la duración de /i/ es menor en comparación con el resto de los valores, presentando incluso tendencia a que la mayoría de sus realizaciones se encuentren bajo la mediana. Esto comprueba la segunda hipótesis planteada en esta investigación, esto es, que existe variación en la duración de /i/ según el valor pragmático que se le imprima al sufijo. Esto no solo se comprueba por los resultados entregados al analizar el valor diminutivo, sino que además por el comportamiento de los otros valores al ser comparados entre ellos.

El valor intensificador se diferencia del valor diminutivo. Sin embargo, no existen evidencias suficientes para afirmar que exista una duración mayor de este valor por sobre los otros; por lo tanto, la aseveración de Alonso (1951) de que es en este valor donde se da un alargamiento en la duración de la vocal y que es precisamente esto lo que permite a los hablantes identificarlo, según los datos, no es del todo acertada.

La intensificación y la atenuación, que son valores totalmente opuestos en su sentido, no presentan diferencias significativas, y de hecho tampoco se diferencian de los otros valores, salvo del diminutivo.

Si bien es cierto, el grupo de sufijos con valor activo mostró ser el promedio de duración más largo, el grado de dispersión de los datos y la dificultad por parte de los participantes de la encuesta para reconocerlo no permiten establecer juicios taxativos sobre su comportamiento. Esto mismo aplica de igual manera al valor afectivo.

Los sufijos apreciativos diminutivos que cumplen el rol reconocido tradicionalmente de dar a entender reducción de tamaño tienen un promedio estadísticamente menor de duración de su vocal tónica con respecto a los otros valores pragmáticos. Existe un comportamiento similar entre algunos grupos estudiados y en otros parece imposible establecer diferencias significativas debido a la dispersión de los datos.

Los resultados muestran que efectivamente existe influencia del valor pragmático en la duración de /i/ en el sufijo diminutivo -ito del español de Chile. Investigaciones anteriores que han estudiado los factores que inciden en la duración de la vocal, como los de Marín (1994-1995) y Hely (19961997), no incluyen el valor pragmático como posible factor a considerar. En el presente estudio, se ha confirmado que la intención comunicativa es un factor que influye en la duración de la vocal acentuada y que la relación entre pragmática y fonética debe ser más considerada a futuro en estudios de este tipo. Esta investigación demuestra que la intención comunicativa puede ser de igual manera un factor a considerar cuando se realiza un estudio sobre la cantidad vocálica del español. 


\section{REFERENCIAS BIBLIOGRÁFICAS}

Alonso, A. 1951. Noción, emoción, acción y fantasía en los diminutivos. Madrid: Gredos.

Fernández Planas, A. M. 2005. Así se habla: nociones fundamentales de fonética general y española. Apuntes de catalán, gallego y euskara. Barcelona: Horsori.

Hely CuencA, M. 1996-1997. Análisis instrumental de la duración de las vocales en español. Philologia Hispalensis 11(1), 295-307.

Herrero, J. 2006. Teorías de pragmática, de la lingüística textual y de análisis del discurso. Cuenca: Ediciones de la Universidad de Castilla-La Mancha.

Hidalgo, A. 2006. Aspectos de la entonación española: viejos y nuevos enfoques. Madrid: Arco Libros.

Hummel, M. 1997. Para la lingüística de vuestro diminutivo: los diminutivos como apreciativo. Anuario de estudios filológicos XX: 191-210.

MARín, R. 1994-1995. La duración vocálica en Español. E.L.U.A 10: 213-226.

Martínez Celdrán, E. 1998. Análisis espectrográfico de los sonidos del habla. Barcelona: Ariel

Pandolfi, A. M. 1989. -illo: ¿Sufijo diminutivo? R.L.A 27: 133-149.

Pereira, D. 2011. Análisis acústico de los marcadores discursivos a ver, bueno, claro, vale, ¿cómo? y ya. Onomázein 24: 85-100.

Pereira, D. y J. Soto-Barba. 2011. Duración absoluta de las vocales del español urbano y rural de la provincia de Ñuble. Boletín de Filología XLVI (1): 153-161.

Quilis, A. y J. Fernández. 1969. Curso de fonética y fonología española. Madrid: Consejo Superior de Investigaciones Científicas.

Quintrileo, C. 2001. Funciones del diminutivo en el español coloquial de Chile. Tesis para optar al grado de Magíster en Lingüística. Universidad de Concepción.

RAE. 2015. Diccionario la Lengua Española. [en línea]. Disponible en http://www.rae.es/ obras-academicas/diccionarios/diccionario-de-la-lengua-espanola.

Reynoso, J. 2005. Procesos de gramaticalización por subjetivización: el uso del diminutivo en español. Selected Proceedings of the 7th Hispanic Linguistics Symposium. Somerville.

RoldÁn, Y. 2000. Correlatos acústicos de actos de habla atenuados del español de Chile. Onomázein 5: 107-118.

Searle, J. 1969. Speech Acts. Cambridge: Cambridge University Press.

Seco, M. 1994. Gramática esencial del español: Introducción al estudio de la Lengua. Madrid: Espasa-Calpe.

Valderrama, R. 2010. Procedimientos técnicos usados en la traducción de sufijos apreciativos diminutivos desde el español de Chile al inglés. Tesina para optar al grado de licenciado en traductología. Universidad de Concepción.

VArela, S. 2005. Morfología léxica: la formación de palabras. Madrid: Gredos. 
Anexo 1. Libreto del protocolo de elicitación del corpus

\section{SCRIPT}

(1)

A: está lindo el día, ¿cierto?

B: ¡Claro! Después de estos días fríos hoy se siente mucho más calentito ${ }^{1}$.

A: Oye, no te había preguntado... ¿Cómo está tu viejo?

$\mathrm{B}$ : Mi viejito ${ }^{2}$ está súper bien, gracias.

A: ¿Qué le pasó?

B: Estuvo un poquito ${ }^{3}$ enfermito $^{4}$, aún no sabemos lo que fue, pero ya puede caminar $\underline{\text { solito }}^{5}$ de nuevo.

A: No sabes cuánto me alegro.

(2)

A: ¿Quieres un tecito ${ }^{6}$, un $\underline{\text { cafecito }}^{7}$, tal vez?

B: No, gracias, estoy bien así... te puedo hacer una pregunta... ¿has visto a $\underline{\text { Jorgito }}^{8}$ ?

A: La verdad es que hace años que no sé nada de él.

B: Mi hermano se ha sentido $\underline{\underline{s o l i t o}}^{9}$ desde que se fue.

A: Me imagino, ellos eran amigos desde chiquititos $^{10}$.

A: Luchito ${ }^{11}$, ¿me pasas esa cajita ${ }^{12}$, por favor?

B: ¿Cuál de las tres quieres?

A: Mira, hay una grande, una mediana y una pequeña. Yo quiero la pequeñita $^{13}$.

B: ¿Ésta?

A: sí... mmm, me parece muy grande. Creo que en el cajoncito ${ }^{14}$ de ahí hay una caja más chiquitita ${ }^{15}$.

B: Aquí no hay nada.

A: Ahora te me quedas calladito ${ }^{16} \mathrm{y}$ harás todas tus tareas.

B: Pero papito ${ }^{17}$, quiero salir a jugar.

A: No, primero quiero toda la loza limpiecita ${ }^{18}$ y tus tareas hechas.

B: Ok,... tengo un problema, acá solo hay esponjas grandes... ¿Dónde están las esponjitas ${ }^{19}$ ?

A: En el cajoncito ${ }^{20}$ chico de al medio. 
(5)

Mis viejos siempre me dicen que estoy gordito ${ }^{21}$ y algo pasadito ${ }^{22}$ de edad para salir a fiestas. Cuando eso sucede me gustaría que se quedaran $\underline{\text { calladitos }}^{23}$ y se metieran en sus asuntos. Ellos no saben que en mi pieza tengo una cajita ${ }^{24}$ donde guardo comida. Sé que ellos se enojan cuando como mucho y que cuando me dicen: "hijo, ¿quieres tortita ${ }^{25}$ ?" lo hacen sólo para saber si caigo en su trampa o no. 
ANEXo 2. Ejemplo de la encuesta de validación del instrumento

\section{Hoja de Respuestas}

Nombre:

Edad:

Sexo: M F

Ocupación:

Lee atentamente las oraciones del documento. En las oraciones encontrará palabras subrayadas, estas contienen sufijos apreciativos. Lea las oraciones en contexto y clasifique al sufijo del 1 al 5 según la que usted cree es la intención del hablante al decirlo. Sólo marque 1 opción.

1) El sufijo sirve para reducir el tamaño físico del objeto

2) El sufijo es utilizado principalmente para dar órdenes, peticiones e invitaciones.

3) El sufijo busca amortiguar algo negativo.

4) El sufijo sirve para establecer un grado de cercanía.

5) El sufijo intensifica el significado de la palabra.

\begin{tabular}{|c|c|c|c|c|c|}
\hline D1 & $\begin{array}{l}1 \\
\bigcirc\end{array}$ & $\begin{array}{l}2 \\
0\end{array}$ & $\begin{array}{l}3 \\
0 \\
\end{array}$ & $\begin{array}{l}4 \\
\bigcirc\end{array}$ & $\begin{array}{l}5 \\
0 \\
\end{array}$ \\
\hline D2 & 0 & 0 & 0 & 0 & 0 \\
\hline D3 & 0 & 0 & 0 & 0 & 0 \\
\hline D4 & 0 & 0 & 0 & 0 & 0 \\
\hline D5 & 0 & 0 & 0 & 0 & 0 \\
\hline D6 & 0 & 0 & 0 & 0 & 0 \\
\hline D7 & 0 & 0 & 0 & 0 & 0 \\
\hline D8 & 0 & 0 & 0 & 0 & 0 \\
\hline D9 & 0 & 0 & 0 & 0 & 0 \\
\hline $\mathrm{D} 10$ & 0 & 0 & $\bigcirc$ & 0 & 0 \\
\hline D11 & 0 & 0 & 0 & 0 & 0 \\
\hline $\mathrm{D} 12$ & 0 & 0 & 0 & 0 & 0 \\
\hline
\end{tabular}




\section{AnEXo 3. Detalle de los resultados de la encuesta de validación del} instrumento

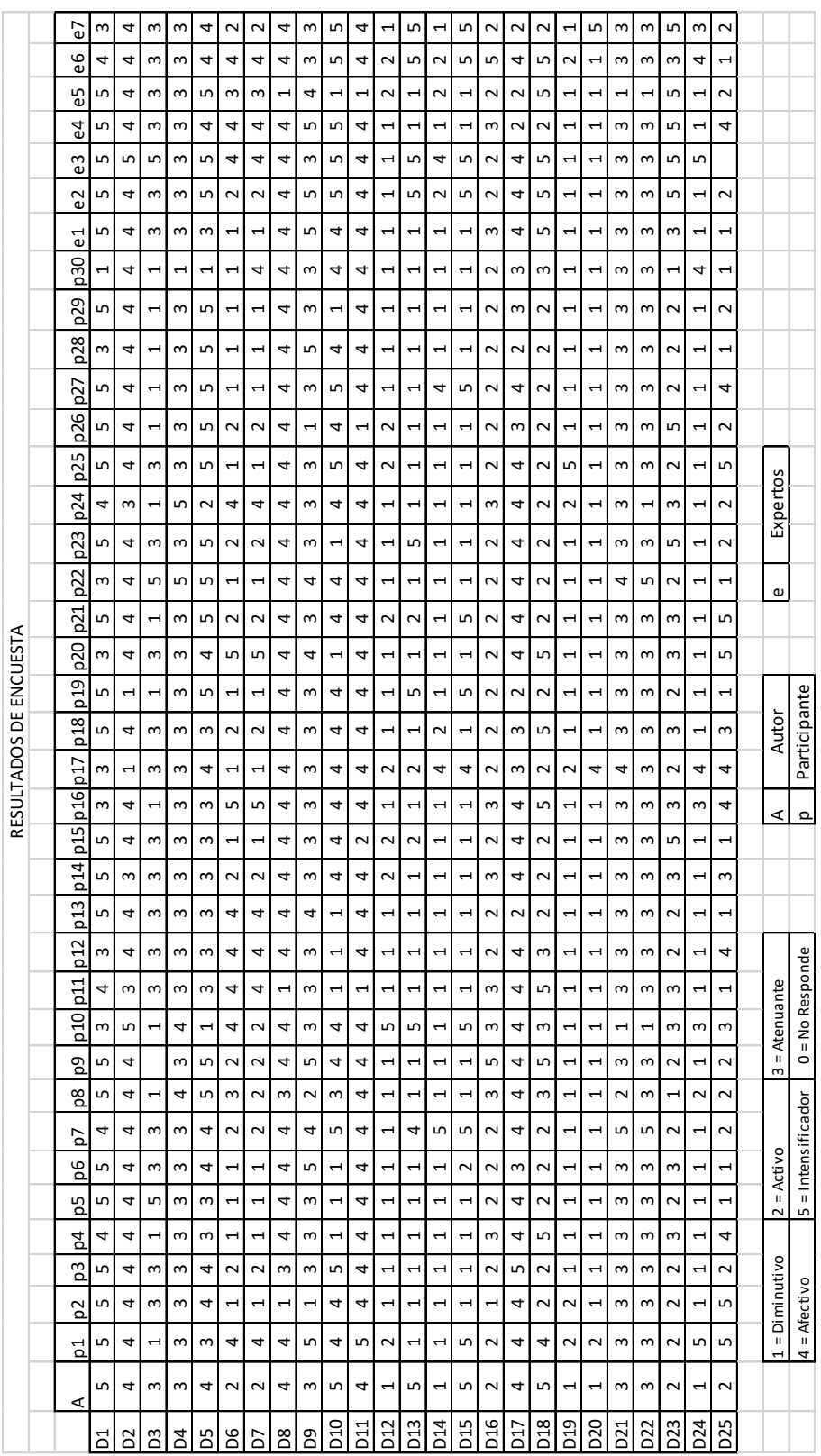

